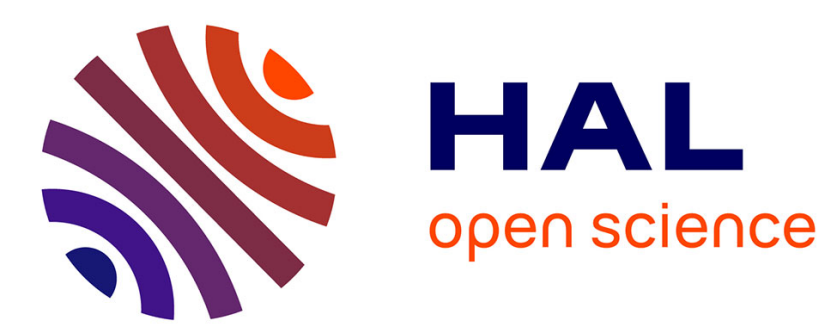

\title{
System identification of photosensitiser uptake kinetics in photodynamic therapy
}

Thierry Bastogne, Loraine Tirand, Muriel Barberi-Heyob, Alain Richard

\section{To cite this version:}

Thierry Bastogne, Loraine Tirand, Muriel Barberi-Heyob, Alain Richard. System identification of photosensitiser uptake kinetics in photodynamic therapy. Sep 2006, pp.CD-ROM. hal-00119433

\section{HAL Id: hal-00119433 \\ https://hal.science/hal-00119433}

Submitted on 9 Dec 2006

HAL is a multi-disciplinary open access archive for the deposit and dissemination of scientific research documents, whether they are published or not. The documents may come from teaching and research institutions in France or abroad, or from public or private research centers.
L'archive ouverte pluridisciplinaire HAL, est destinée au dépôt et à la diffusion de documents scientifiques de niveau recherche, publiés ou non, émanant des établissements d'enseignement et de recherche français ou étrangers, des laboratoires publics ou privés. 


\title{
SYSTEM IDENTIFICATION OF PHOTOSENSITISER UPTAKE KINETICS IN PHOTODYNAMIC THERAPY
}

\author{
T. Bastogne ${ }^{*}$ L. Tirand ${ }^{* *}$ M. Barberi-Heyob ${ }^{* *}$ A. Richard R $^{*}$ \\ * Université Henri Poincaré, Nancy 1, \\ Centre de Recherche en Automatique de Nancy (CRAN), \\ CNRS-INPL-UHP UMR 7039, \\ BP 239, F-54506 Vandouvre-lès-Nancy Cedex, France \\ ${ }^{* *}$ Centre Alexis Vautrin, Centre de Lutte contre le Cancer \\ Brabois, Av. de Bourgogne, 54511 Vandœuvre-lès-Nancy Cedex
}

\begin{abstract}
This study presents the contributions of system identification techniques to the experimental modelling of photosensitiser uptake kinetics in photodynamic therapy. The experimental framework is limited to one cancer cell line (HT29-A4), one photosensitiser (Chlorin e6), one photosensitiser dose $\left(5 \mu \mathrm{g} \cdot \mathrm{ml}^{-1}\right)$, four albumin rates in a monolayer culture and eight measurements between $1 h$ and $24 h$. Issues associated with this experimental modelling study are the deficiency of measurement points, low signal-to-noise ratios and 'poor' excitation signals (step signals). This paper deals with model structure selection, parameter estimation and parameter uncertainty description by probabilistic confidence regions. Moreover, an explicit relationship between the static gain of the estimated model and the albumin rate of the culture medium has been established *
\end{abstract}

* Presented at the 6th IFAC Symposium on Modelling and Control in Biomedical System (including biological systems), Reims, France, September 2006

Keywords: system identification, pharmacokinetics, drug delivery, photodynamic therapy

\section{INTRODUCTION}

Photodynamic therapy (PDT) (Moser, 1998) is an emerging therapy for displastic tissues such as cancers. This therapy involves selective uptake and retention of a photosensitive drug (photosensitiser, PS) in a tumour, followed by irradiation with light at an appropriate wavelength. The activated photosensitiser is thought to produce singlet oxygen at high doses and thereby to initiate apoptotic and necrotic death of tumour. In current clinical practice, photodynamic therapy is carried out with prescribed drug doses and light doses as well as fixed drug-light intervals and illumination fluence rates. These doses are determined from a physical model, see e.g. (Patterson et al., 1990; Hetzel et al., 2005), defined in equation (1).

$$
[R]=k_{s} \cdot b \cdot \epsilon \cdot I_{\lambda} \cdot T \cdot\left[{ }^{1} P\right] \cdot \Phi \cdot f
$$

where: $[R]$ is a threshold concentration of oxidising events radicals that needs to occur in a sensitive location within a cancer cell to elicit the cascade toward cell death, $I_{\lambda}$ is the irradiance on the tissue surface, $T$ is the exposure time of treatment light and $\left[{ }^{1} P\right]$ is the concentration of intracellular photosensitive drug (photosensitiser $\mathrm{P}$ ). $k_{s}$ is the backscatter factor due to reflected light from underlying tissue, $b$ is a conversion factor, $\epsilon$ is the extinction coefficient of photosensitive drug, $\Phi$ is the quantum yield for conversion of activated drug to oxidising radicals, which usually depends on the oxygen concentration dissolved in the cells and $f$ is the fraction of generated oxidising radicals, which attack sensitive cellular sites, while the fraction $(1-f)$ of the radicals attack lesser 
sites and have minor effect. Despite its current use in clinical applications, several polemical points can be addressed against this model:

- Equation (1) shows a simple reciprocity of photosensitiser concentration and light. Nevertheless, several experiments have shown contradictory results (Moesta et al., 1995; Yuan et al., 1997). Moreover Potter et al. have shown that a reduction in photosensitiser concentration during treatment, e.g. PS photodegradation, is an important consideration (Potter, 1986) .

- The term $(\Phi)$ is function of oxygenation but is usually a unknown factor during PDT (Tromberg et al., 1990; Nichols and Foster, 1994; Hetzel et al., 2005; Dysart et al., 2005).

- Sites of photodamage mainly depend on the location of the PS in the cell. Sites of action for singlet oxygen in PDT include mitochondria, endoplasmic reticulum, Golgi apparatus, lysosomes, DNA and lipid membranes (Henderson and Dougherty, 1992; Epe et al., 1993). Some of them are critical sites. Unfortunately, equation (1) does not take into account the intracellular location of PS.

- In fact, all quantities are generally time dependent, i.e. their concentrations (or in the case of light, its fluence rate) can change during irradiation. In other words, PDT is a dynamic process but equation (1) is just a static model (Georgakoudi et al., 1997; Georgakoudi and Foster, 1998; Dysart et al., 2005).

This previous list is not exhaustive but presents some of the main modelling requirements and challenges for increasing scientific knowledge of PDT. System identification (Ljung, 1987; Söderström and Stoica, 1989; Walter and Pronzato, 1997) is the field of mathematical modelling of dynamic systems from experimental data. No identification study has ever been applied to the PDT problem. It is therefore interesting to assess the contributions of system identification to the experimental modelling of PDT.

The problem addressed in this paper deals with system identification of in vitrophotosensitiser uptake kinetics, i.e. the first phase of PDT. Problems encountered are generic problems in biology, i.e few measurement points $\left(n_{e} \cdot n_{t}<20\right)$, low signal-to-noise ratios due to a great measurement variability and 'poor' stimulus signals (step signals in general). In this experimental framework, objectives are : (1) selecting a model structure $(\mathcal{M}(\mathbf{p})),(2)$ estimating its parameters $(\mathbf{p})$, (3) determining the parameter uncertainties and if necessary explaining how the serum concentration in the culture medium $([S e])$ can influence model parameters, i.e. determining the relationship $f(\cdot)$ such that $\hat{\mathbf{p}}=f([S e])$.

This paper is organised as follows. A macroscopic model of PDT is firstly presented in section 2 to introduce the system identification problem of PS uptake kinetics under a restricting experimental framework. The remaining sections are put into a chronological order of system identification steps. Section 3 deals with model structure selection, section 4 presents the estimation step, section 5 describes the parameter uncertainty, section 6 concerns the validation of the noise hypothesis and section 7 discusses on the explicit influence of the serum concentration.

\section{MATHEMATICAL MODEL OF PDT}

\subsection{Macroscopic model}

Table 1. Main notations

\begin{tabular}{llr}
\hline Symb. & Description & $M$ \\
\hline$[x]$ & concentration of & $m o l$ \\
$Q_{x}$ & quantity of $x$ & $M \cdot s$ \\
$D_{x}$ & dose of $x$ & $s$ \\
$t$ & time & \\
$P$ & photosensitiser molecule & \\
$P_{a}$ & administrated PS & \\
$P_{i}$ & intracellular PS & \\
$P_{x}$ & extracellular PS & \\
$P_{P P}$ & photoproduct of PS & \\
$z_{P}$ & photosensitiser intraC. colocation & A.U. cell ${ }^{-1}$ \\
$M$ & Medium & \\
$S e$ & serum (proteins) in the medium & \\
$C C L$ & cancer cell line & \\
$A_{t h}$ & critical intracellular damage threshold & \\
$L$ & light & \\
$I_{\lambda}$ & fluence rate of light with wavelength $\lambda$ & $W \cdot m^{-2}$ \\
$S$ & cancer cell survival rate & \\
\hline $\mathbf{p}$ & parameter vector of a model & \\
$\mathcal{M}(\cdot)$ & model structure & \\
$s$ & differentiation operator & \\
$n_{t}$ & number of measurement points & \\
$n_{e}$ & number of repeated experiments & \\
$S N R$ & signal-to-noise ratio & \\
\hline
\end{tabular}

A macroscopic model of PDT is described by a block diagram in figure 1. Main variables, except the standard notations of chemical elements, are given in table 1. This macroscopic model is composed of three macro-blocks associated with the three main phases of PDT, i.e. the PS uptake, the irradiation and the cytotoxicity phases. The photosensitiser, light generator, cell culture medium and cancer cell line are described by signal generators (grey blocks). The main input variables, $I_{\lambda}, Q_{P a}$ and $\left[\mathrm{O}_{2}\right]$ are represented by bold arrows. $\left[S_{e}\right]$ and $C C L$ are usually supposed to be constant during the therapy. $\lambda$ is regarded as a parameter of the light source. The PS uptake and cytotoxicity phases are described by black-box models since their dynamic behaviour is not well understood currently. $e_{P}, e_{\left[\mathrm{O}_{2}\right]}, e_{S}$ are error variables describing the combined effects of modelling errors, measurement noises and disturbances.

\subsection{PS uptake kinetics}

A sketch of the PS uptake phenomenon under in vitro condition is shown in figure 2. During this phase, a mass balance equation applied to the PS molecules is: 


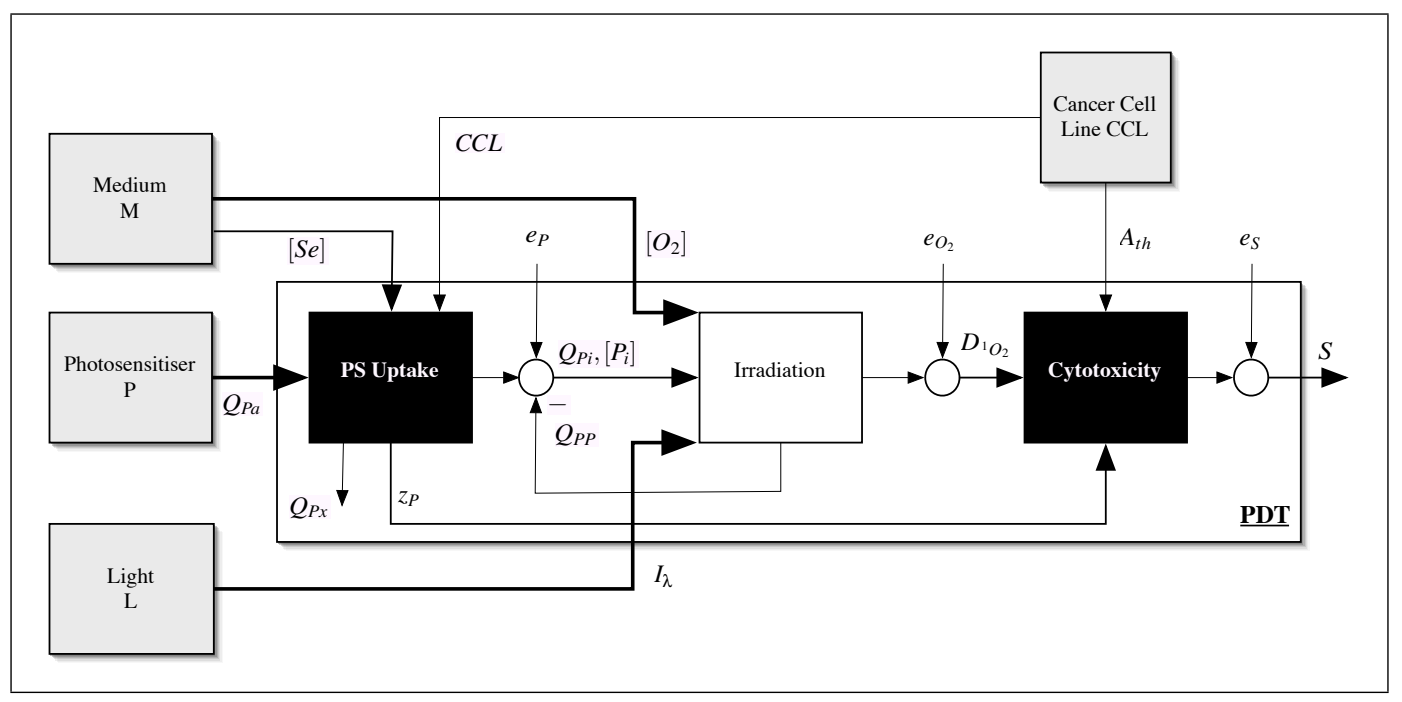

Figure 1. Block diagram of the PDT

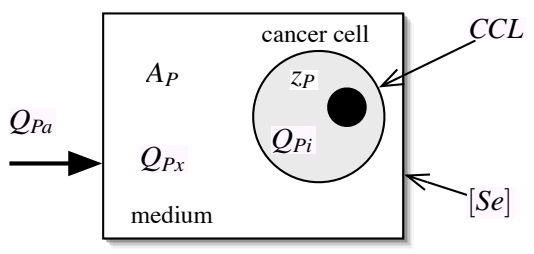

Figure 2. PS uptake phenonenon

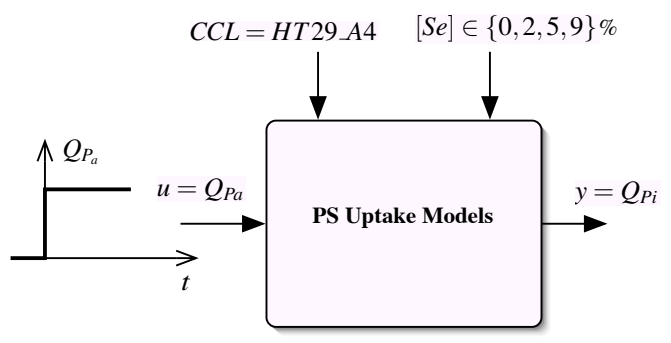

Figure 3. Experimental protocol

$$
Q_{P a}(t)=Q_{P i}(t)+Q_{P x}(t)
$$

The uptake kinetics between the input variable $Q_{P a}(t)$ and the output variable $Q_{P i}(t)$ is described as follows:

$$
Q_{P i}(t)=\mathcal{M}\left(t, \mathbf{p}, Q_{P a}\right)+e(t)
$$

where the model structure $\mathcal{M}(\cdot)$ and its parameters p are unknown. $e(t)$ corresponds to the output error, i.e. the error between the measured output $\left(Q_{P i}(t)\right)$ and the model output $\left(\hat{Q}_{P_{i}}(t)=\mathcal{M}\left(t, \mathbf{p}, Q_{P a}\right)\right) . e(t)$ is assumed to be a random variable with a normal distribution defined by $\pi_{e}(e(t)) \propto \mathcal{N}\left(0, \sigma_{e}^{2}\right)$. The sequence $\mathbf{e}=\left(e\left(t_{1}\right) \cdots e\left(t_{n_{t}}\right)\right)$ is supposed to be independent and identically distributed.

\subsection{Experimental data}

Materials and methods concerning PS uptake experiments are described in (Barberi-Heyob et al., 2004). The experimental protocol is summarised in figure 3 . A step signal was applied to $Q_{P a}$ (step magnitude
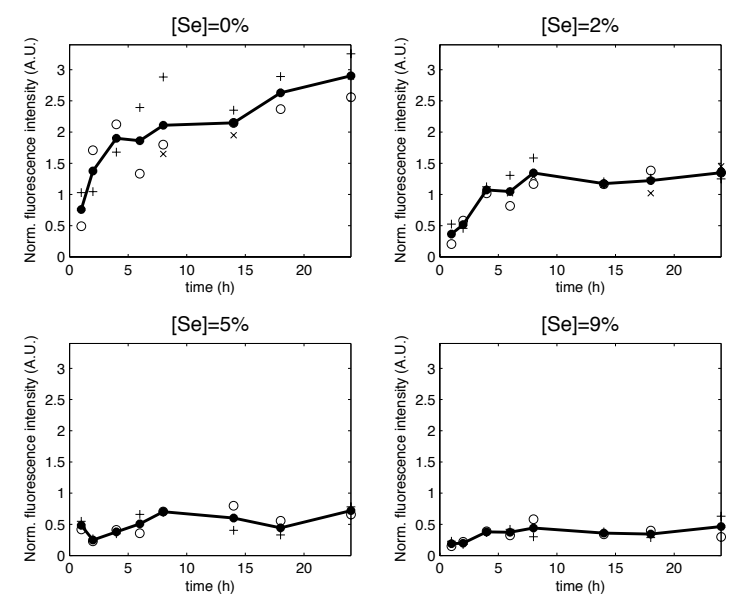

Figure 4. Four estimation data sets

$\left.5 \mu \mathrm{g} \cdot \mathrm{ml}^{-1}\right) . C C L$ is a human colon cancer cell line (HT29A4) and the PS molecule $(P)$ is chlorin e6 (Ce6). Four experiments were carried out for four different doses of albumin $([S e] \in\{0,2,5,9 \% B S A\})$ where BSA denotes bovine serum albumin. The four responses of $Q_{P i}$, measured with spectrofluorimeter, are shown in figure 4. Measurement times are defined

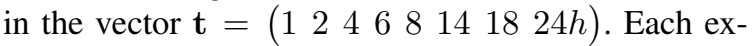
periment was repeated two or three times and the full line plot corresponds the mean response. Note that the variation between two samples measured at the same time can reach about $66 \%$, i.e. a signal-to-noise ratio estimated to $R S B \approx 3,5 d B$.

\section{SELECTION OF A KINETIC MODEL STRUCTURE}

The first question deals with the determination of a parsimonious model structure $(\mathcal{M}(\mathbf{p}))$ among a set $\mathbb{M}$ of candidate model structures. Three a priori distinguishable model structures (Walter and Pronzato, 1997) are examined, $\mathbb{M}=\left\{\mathcal{M}_{1}, \mathcal{M}_{2}, \mathcal{M}_{3}\right\}$ with:

$\mathcal{M}_{1}:\left(1+T_{1} \cdot s\right) Q_{P i}(t)=k_{1} \cdot Q_{P a}(t)$ 


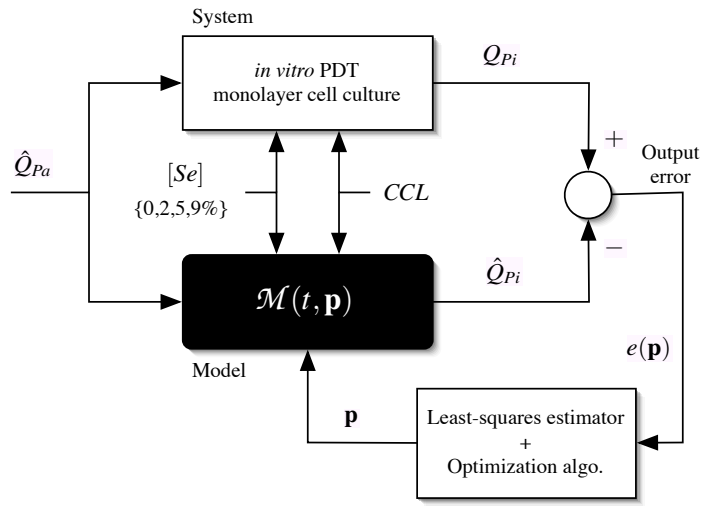

Figure 5. Output error method for a parallel model

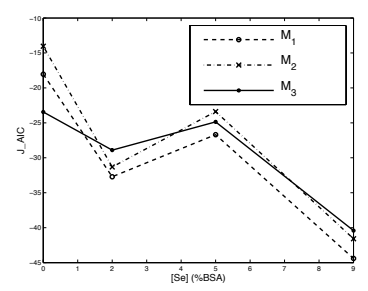

(a) $\mathrm{AIC}$

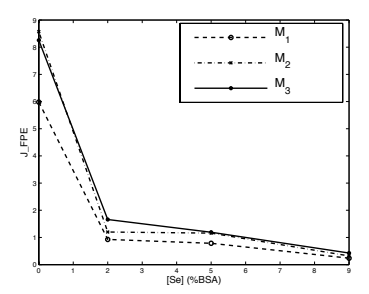

(b) FPE
Figure 6. Comparison of model structures

$\mathcal{M}_{2}:\left(1+T_{21} \cdot s\right)\left(1+T_{22} \cdot s\right) Q_{P i}(t)=k_{2} \cdot Q_{P a}(t) ;$

$\mathcal{M}_{3}:\left(1+T_{31} \cdot s\right)\left(1+T_{32} \cdot s\right) Q_{P i}(t)=k_{3} \cdot\left(1+T_{33} \cdot s\right) Q_{P a}(t)$.

$s$ is the differentiation operator defined by: $s \cdot Q_{P}(t) \stackrel{\text { def }}{=}$ $\partial Q_{P} / \partial t$. For each model structure $\mathcal{M}_{i}$ is associated a parameter vector $\mathbf{p}_{i}$ belonging to some prior feasible set $\mathbb{P}_{i}$. In this application, the Akaike's AIC criterion, defined in equation (4) is used. For a synoptic presentation of various available criteria of model structures, see e.g. (Söderström, 1977).

$$
\widehat{\mathcal{M}}(\hat{\mathbf{p}})=\arg \min _{\mathcal{M}_{i} \in \mathbb{M}} \min _{\mathbf{p}_{i} \in \mathbb{P}_{i}} J_{A I C}\left(\mathcal{M}_{i}\left(\mathbf{p}_{i}\right), t\right),
$$

with $J_{A I C}=1 / n_{t}\left(-\ln \left(\pi_{\mathbf{Q}_{P i}}\left(\mathbf{Q}_{P i}^{k} \mid \mathbf{p}_{i}\right)\right)+\operatorname{dim}\left(\mathbf{p}_{i}\right)\right)$, $\mathbf{Q}_{P i}=\left(Q_{P i}\left(t_{1}\right) \cdots Q_{P i}\left(t_{n_{t}}\right)\right)$ and $\mathbf{Q}_{P i}^{k}$ is the $k^{t h}$ realisation of the output measurement vector $\mathbf{Q}_{P i}$. By taking into account the probabilistic distribution of output errors (see paragraph 2.2), minimising $J_{A I C}$ comes to minimise:

$$
\tilde{J}_{A I C}=n_{t} \cdot \ln \left(\sigma_{e}^{2}\right)+2 \operatorname{dim}\left(\mathbf{p}_{i}\right)
$$

where the variance $\sigma_{e}^{2}$ is a function of $\mathcal{M}_{i}\left(\mathbf{p}_{i}\right)$. For each model structure, $\hat{\mathbf{p}}_{i}$ is obtained by a non linear least squares estimator, as shown in figure 5. Results of the model structure selection are gathered in figure 6 . The most parsimonious model structure corresponds to $\mathcal{M}_{1}$ since it minimises $J_{A I C}$ for three different values of $[S e]$. As illustrated in figure 6(b), this choice is confirmed by the final prediction error (FPE) criterion.

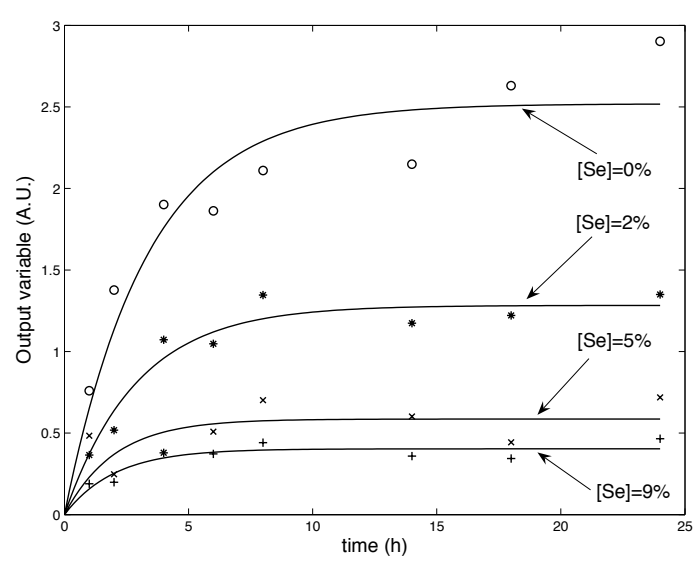

Figure 7. Measured and simulated responses of $Q_{P i}$

\section{PARAMETER ESTIMATION}

The parameter estimation relies on the output error method for a parallel model as shown in figure 5 in which the optimisation step is based on a Levenberg Marquardt algorithm. Estimates of $\mathbf{p}_{1}=\left(k_{1} T_{1}\right)$ are given in table 2 . Figure 7 compares the measured (mean values) and simulated step responses of $Q_{P i}$ for the four different albumin rates.

Table 2. Estimation results

\begin{tabular}{lllll}
\hline$[\mathrm{Se}]$ & $0 \%$ & $2 \%$ & $5 \%$ & $9 \%$ \\
\hline \hline$k_{1}$ & 501 & 257 & 117 & 81 \\
\hline$T_{1}(h)$ & 3.19 & 2.93 & 2.02 & 2.03 \\
\hline
\end{tabular}

\section{PARAMETER UNCERTAINTY}

Determining the optimal value of the parameters with respect to a chosen criterion is not enough. It is also important to evaluate the uncertainty associated with those estimates. Herein, parameters uncertainty is described by confidence regions, noted $\mathcal{R}^{\alpha}$, defined in (Hamilton et al., 1982; Walter and Pronzato, 1997) as follows:

$\mathcal{R}^{\alpha}=\left\{\mathbf{p} \in \mathbb{R}^{n_{p}} \mid \frac{e^{T}(\mathbf{p}) \Pi(\mathbf{p}) e(\mathbf{p})}{n_{p} \hat{\sigma}^{2}} \leq F_{\alpha}\left(n_{p}, n_{e}-1\right)\right\}$

$\mathcal{R}^{\alpha}$ defines a $100(1-\alpha) \%$ confidence region for the parameters. $F_{\alpha}\left(n_{1}, n_{2}\right)$ denotes a Fisher-Snedecor distribution with $n_{1}$ and $n_{2}$ degrees of freedom. $\mathbf{e}(\mathbf{p})=\mathbf{Q}_{P i}-\hat{\mathbf{Q}}_{P i}(\mathbf{p})$ is the output error vector $\epsilon$ $\mathbb{R}^{n_{t}}$. The orthogonal projection matrix $\Pi(\mathbf{p})$ is given by:

$$
\Pi(\mathbf{p})=S_{\hat{\mathbf{Q}}_{P i}}\left(S_{\hat{\mathbf{Q}}_{P i}}^{T} \cdot S_{\hat{\mathbf{Q}}_{P i}}\right)^{-1} S_{\hat{\mathbf{Q}}_{P i}}^{T},
$$

where $S_{\hat{\mathbf{Q}}_{P i}}=\partial \hat{\mathbf{Q}}_{P i}(\mathbf{p}) / \partial \mathbf{p}^{T}$ is the sensitivity function (gradient) of the output model in respect with the parameter vector. The estimated noise variance is given by: 


$$
\begin{aligned}
\hat{\sigma}^{2} & =\frac{1}{n_{e} n_{t}-1} \sum_{i=1}^{n_{t}} \sum_{k=1}^{n_{e}}\left(Q_{P i}\left(t_{i, k}\right)-\bar{Q}_{P i, i}\right)^{2} \\
\bar{Q}_{P i, i} & =\frac{1}{n_{e}} \sum_{k=1}^{n_{e}} Q_{P i}\left(t_{i, k}\right) .
\end{aligned}
$$

$n_{e}$ refers to the number of repeated experiments. Figure 8 shows the four $95 \%$ confidence regions associated with the four values of the albumin rate. This result reveals a large uncertainty about the estimates, mainly for $\hat{T}_{1}$. The number of time instants cannot be significantly increased in practice. Nevertheless, adapting materials and measurement systems in order to increase the number of samples at each time instant is possible. This solution could significantly reduce the area of confidence regions.

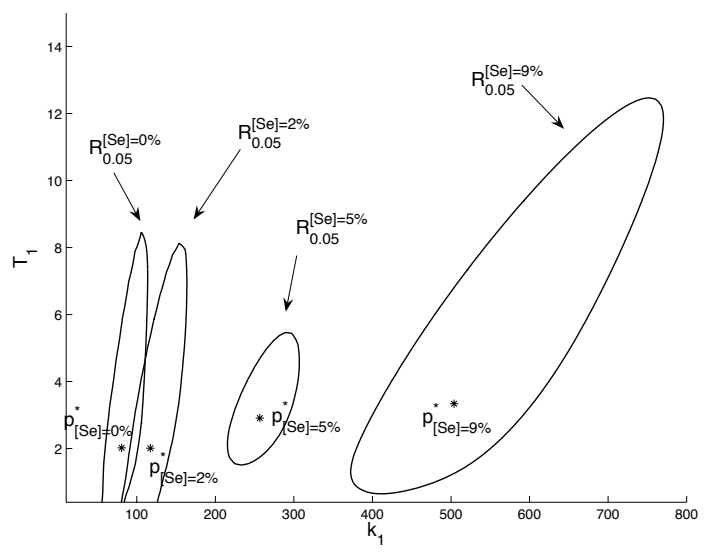

Figure $8.95 \%$ confidence regions for $\hat{k}_{1}$ and $\hat{T}_{1}$

\section{NOISE HYPOTHESIS VALIDATION}

The determination of the confidence regions relies on the assumption that $\left\{e\left(t_{i}, \mathbf{p}\right)\right\}$ is a sequence of normal random variables, independent and identically distributed, i.e. $e\left(t_{i}, \mathbf{p}\right) \propto \mathcal{N}\left(0, \sigma_{e}^{2}\right)$. Histograms of output residuals are depicted in figure 9 . Their empirical distribution is clearly dependent on the serum rates ([Se]). Unfortunately, the number of realisations is too small $(\leq 20)$ to accurately check the Gaussian distribution of the residuals. However, the assumption about the nullity of the mean is perceptibly valid. Moreover, almost all histograms are symmetric. There is thereby no convincing reason to reject the initial hypothesis. In other terms, no conclusion can be drawn about the validity of the noise assumption. In fact, in this experimental setup, the gaussian and i.i.d. assumption, usual stochastic paradigm in system identification, is not suited to the description of the parameter uncertainty. In perspective, it would be interesting to assess recent alternative approaches like the interval analysis (Jaulin et al., 2001) or the Leave-out Sign-dominant Correlation Regions proposed by Campi and Weyer in (Campi and Weyer, 2006).

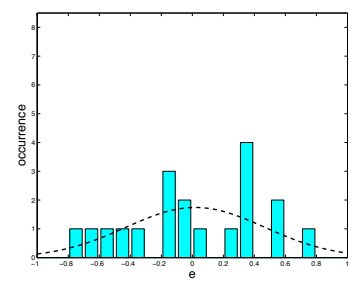

(a) $[\mathrm{Se}]=0 \%, m_{e} \approx 1.9$. $10^{-2}, \sigma_{e} \approx 0.45$

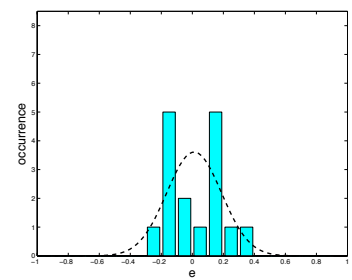

(c) $[S e]=5 \%, m_{e_{r}} \approx 1.1$. $10^{-2}, \sigma_{e_{r}} \approx 0.18$

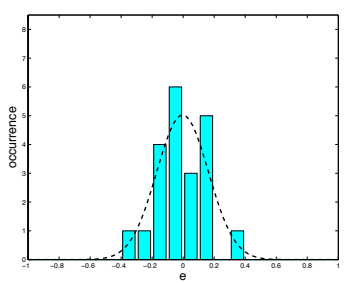

(b) $[S e]=2 \%, m_{e} \approx 3.7$ $10^{-3}, \sigma_{e} \approx 0.17$

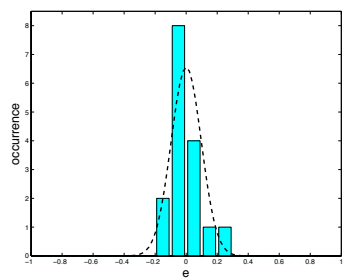

(d) $[S e]=9 \%, m_{e} \approx 4.2$. $10^{-4}, \sigma_{e} \approx 9.7 \cdot 10^{-2}$
Figure 9. Histograms of output residuals for $\mathcal{M}_{1}$

\section{ANALYSIS OF THE SERUM DEPENDANCE}

Figure 10(a) shows that $\hat{k}_{1}$ is a decreasing function of $[S e]$. The dotted graph refers to the four estimates of $k_{1}$ and the continuous curve is a regression model, defined in equation (9).

$$
\hat{k}_{1} \approx \frac{922}{[S e]+1.83} .
$$

This model expresses an inversely proportional relationship between the static gain $\hat{k}_{1}$ and the serum rate $[S e]$. A new in vitro model (monolayer culture) of the PS uptake kinetic, defined in equation 10, can thereby be put forward.

$$
T_{1} \dot{Q}_{P i}(t)+Q_{P i}(t)=\frac{\alpha}{[S e]+\beta} \cdot Q_{P a}(t)+e(t),
$$

where the parameters $T_{1}, \alpha$ and $\beta$ are functions of the cancer cell line. The output error $e(t)$ is supposed to be an independent stochastic process normally distributed $\mathcal{N}\left(0, \sigma^{2}\right)$. No significant relationship has been pointed out between $\hat{T}_{1}$ and $[S e]$.

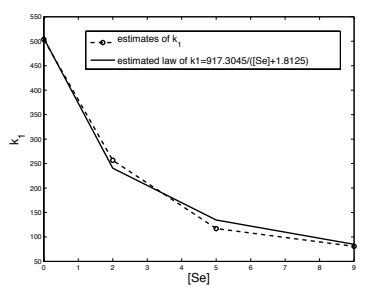

(a) $C C L=H T 29 \_A 4$

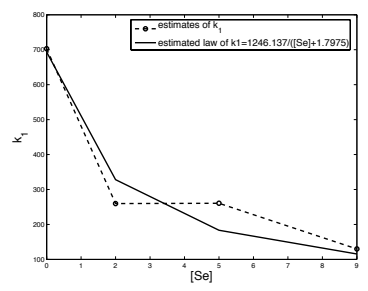

(b) $C C L=H T 29 \_B L K$
Figure 10. Relationship between $k_{1}$ and $[S e]$ for two different $C C L$ 


\section{MODEL VALIDATION}

The previous model has not been cross validated yet but a similar model, i.e. a first-order model, has been identified for another cancer cell line (HT29_BLK). Ffigure 10(b) corobore the inversely proportional relationship between $k_{1}$ and $\left[S e\right.$ ] for the $H T 29_{-} B L K$ line.

\section{CONCLUSION}

Contributions of system identification to the in vitro modelling of photosensitiser uptake kinetics in photodynamic therapy are examined in this study. Difficulties of such an application are triple: (i) lack of measurement, (ii) low signal-to-noise ratio and (iii) 'poor' stimulus signals. The identification procedure deals with model structure selection, parameter estimation, uncertainty description and model validation. The resulting behavioural model relies on a first-order differential equation taking into account the effects of the protein factor. This result has confirmed the applicability of system identification algorithms in such an experimental setup. However, estimates are characterized by large confidence regions. The number of time instants cannot be significantly increased in practice. Nevertheless, adapting materials and measurement systems in order to increase the number of samples at each time instant is possible. Future experiments will be carried out to check the validity of the proposed behavioural model for other photosensitisers and cancer cell lines.

\section{REFERENCES}

Barberi-Heyob, M., P.-O. Védrine, J.-L. Merlin, R. Millon, J. Abecassis, M.-F. Poupon and F. Guillemin (2004). Wild-type p53 gene transfer into mutated p53 HT29 cells improves sensitivity to photodynamic therapy via induction of apoptosis. Int. J. Oncol. (24), 951-958.

Campi, M. C. and E. Weyer (2006). Identification with finitely many data points: The lscr approach. In: Proc. of the 14th IFAC Symposium on System Identification (SYSID).

Dysart, J. S., G. Singh and M. Patterson (2005). Calculation of singlet oxygen dose from photosentitizer fluorescence and photobleaching during mTHPC photodynamic therapy of MLL cells. Photochem. Photobiol. (81), 196-205.

Epe, B., M. Pflaum and S. Boiteux (1993). DNA damage induced by photosensitizers in cellular and cell-free systems. Mutat. Res. 299, 135-145.

Georgakoudi, I. and T. H. Foster (1998). Singlet oxygen- versus nonsinglet oxygen-mediated mechanisms of sensitizer photobleaching and their effects on photodynamic dosimetry. Photochem. photobiol. 67(6), 612-625.
Georgakoudi, I., M. G. Nichols and T. H. Foster (1997). The mechanism of photofrin ${ }^{(c)}$ photobleaching and its consequences for photodynamic dosimetry. Photochem. Photobiol. 65(1), 135-144.

Hamilton, D. C., D. G. Watts and D. M. Bates (1982). Accounting for intrinsic nonlinearities in nonlinear regression parameter inference regions. The Annals of Stat. 10, 386-393.

Henderson, B. W. and T. J. Dougherty (1992). How does photodynamic therapy work?. Photochem. Photobiol. 55, 145-157.

Hetzel, F. W., S. M. Brahmavar, Q. Chen, S. L. Jacques, M. S. Patterson, B. C. Wilson and T. C. Zhu (2005). Photodynamic therapy dosimetry. AAPM report No.88. American Association of Physicists in Medicine by Medical Physics Publishing.

Jaulin, L., M. Kieffer, O. Didrit and E. Walter (2001). Applied Interval Analysis. Springer.

Ljung, L. (1987). System identification: theory for the user. Prentice Hall. Englewood Cliffs, NJ.

Moesta, K. T., W. R. Greco, S. O. Nurse-Finlay, J. C. Parsons and T. S. Mang (1995). Lack of reciprocity in drug and light dose dependence of photodynamic therapy of pancreatic adenocarcinoma in vitro. Cancer Res. 55(14), 3078-3084.

Moser, J. G. (1998). Photodynamic Tumor Therapy: 2nd and 3rd Generation. Gordon \& Breach Science Publishers.

Nichols, M. G. and T. H. Foster (1994). Oxygen diffusion and reaction kinetics in photodynamic therapy of multicell tumor spheroids. Phys. Med. Biol.

Patterson, M.S., B.C. Wilson and R. Graff (1990). In vivo tests of the concept of photodynamic threshold dose in normal rat liver photosensitized by aluminum chlorosulphonated phthalocyanine. Photochem. Photobiol. 51, 343-349.

Potter, W. R. (1986). The theory of photodynamic therapy dosimetry: consequences of photodestruction of sensitizer. Lasers Med. 712, 124129.

Söderström, T. (1977). On model structure testing in system identification. Int. J. Control 26, 1-18.

Söderström, T. and P. Stoica (1989). System identification. University Press, Cambridge, Prentice Hall.

Tromberg, B. J., A. Orenstein, S. Kimel, S. J. Barker, J. Hyatt, J. S. Nelson and M. W. Berns (1990). In vivo oxygen tension measurements for the evaluation of the efficiency of photodynamic therapy. Photochem. Photobiol. 52, 375-385.

Walter, E. and L. Pronzato (1997). Identification of Parametric Models from experimental data. Springer, Masson.

Yuan, J., P. A. Mahama-Relue, R. L. Fournier and J. A. Hampton (1997). Predictions of mathematical models of tissue oxygenation and generation of singlet oxygen during photodynamic therapy. Radiat. Res. 148, 386-394. 